\title{
La Décade et la « colonisation nouvelle »
}

\section{Bernard Gainot}

\section{OpenEdition}

\section{Journals}

\section{Édition électronique}

URL : https://journals.openedition.org/ahrf/2137

DOI : 10.4000/ahrf.2137

ISSN : 1952-403X

\section{Éditeur :}

Armand Colin, Société des études robespierristes

\section{Édition imprimée}

Date de publication : 1 mars 2005

Pagination : $99-116$

ISSN : 0003-4436

\section{Référence électronique}

Bernard Gainot, "La Décade et la « colonisation nouvelle » ». Annales historiques de la Révolution

française [En ligne], 339 | janvier-mars 2005, mis en ligne le 27 avril 2006, consulté le 23 avril 2022.

URL : http://journals.openedition.org/ahrf/2137 ; DOI : https://doi.org/10.4000/ahrf.2137

Ce document a été généré automatiquement le 23 avril 2022.

Tous droits réservés 


\title{
La Décade et la « colonisation nouvelle »
}

\author{
Bernard Gainot
}

1 Le thème abordé est pratiquement passé sous silence dans les études classiques sur La Décade philosophique, littéraire, et politique, que ce soit l'essai de Joanna Kitchin - qui ne fait jamais référence à la thématique de l'abolition et de la colonisation ${ }^{1}$ - ou la thèse de Marc Regaldo - pour qui cette thématique est marginale ${ }^{2}$.

2 Il n'est évidemment pas question de nier le très grand apport de ces synthèses classiques. Je pense néanmoins que les deux faces de la question coloniale entre 1794 et 1802, à savoir la défense du décret d'abolition de l'esclavage du 16 pluviôse an II, et la réflexion concomitante sur l'avenir des établissements d'outre-mer, sont au centre du projet politique défendu par les rédacteurs de La Décade. Je m'appuie, pour ce faire, sur quelques ouvrages récents; avant tout, les études pionnières d'Yves Bénot, et singulièrement son étude fouillée des positions des rédacteurs de La Décade sur le problème de l'esclavage entre 1795 et 1802 dans La démence coloniale sous Napoléon ${ }^{3}$. Je me contenterai bien souvent de renvoyer à ces analyses pour des informations complémentaires, notamment pour tout ce qui concerne la défense du principe abolitionniste, mon propos portant plus spécifiquement sur les contradictions inhérentes aux projets de développement des établissements coloniaux. Il faut encore citer l'ouvrage de Claude Wanquet, La France et la première abolition de l'esclavage, 1794 $1802{ }^{4}$. Cet ouvrage étudie en détail l'évolution des colonies orientales (île Maurice, Réunion), mais il accorde également une large place aux hésitations et contradictions de la politique officielle de la Première République, parallèles aux interrogations et nuances d'opinions que l'on retrouve dans $L a$ Décade, ce qui n'est guère surprenant lorsque l'on repart du projet politique affirmé de cette dernière, comme nous le verrons. Enfin, je dois préciser que cette communication est largement le produit des recherches effectuées sur la seconde société des Amis des Noirs. Le résultat de ces recherches est exposé dans un ouvrage publié en 1998, en collaboration avec Marcel Dorigny ${ }^{5}$. Il montre le rôle éminent joué par plusieurs auteurs de La Décade, dont il sera essentiellement question dans cet article- Jean-Baptiste Say, Charles-Guillaume 
Théremin, Helen-Maria Williams - parmi les dirigeants de cette société abolitionniste, en même temps que l'évidente continuité avec les thématiques développées par la première Société des Amis des Noirs, celle des débuts de la Révolution, dont les registres sont analysés par Marcel Dorigny.

Pourquoi la question coloniale est-elle une question centrale? Il faut renvoyer ici brièvement au projet politique de La Décade. Celui-ci peut être défini comme "républicain conservateur", le terme conservateur n'ayant pas le sens actuel, qui privilégie plutôt la défense d'un modèle de société : à l'époque, il s'agit avant tout de la conservation des institutions républicaines ${ }^{6}$. La préservation et la consolidation de ces institutions sont plus essentielles que la Constitution. En même temps, le bon fonctionnement de cette dernière dépend de l'enracinement des premières ${ }^{7}$. Tout laisse à penser que la liberté générale, c'est-à-dire le décret d'abolition de l'esclavage, indépendamment de toute considération sur les circonstances qui ont entraîné la promulgation de ce décret, figure parmi les institutions républicaines à conserver.

4 Les rédacteurs de La Décade ne sont d'ailleurs pas les seuls à exprimer cette orientation; il suffira de mentionner d'autres journaux comme Le Conservateur de Garat, Daunou, Cabanis, François de Neufchateau, ou bien le Journal d'Économie politique de Roederer ${ }^{8}$. La stratégie est identique : agir comme groupe de pression, comme laboratoire d'idées, avec pour ambition de tenir le rôle de "cerveau" de la Première République. Mais aussi contribuer au débat public, en adoptant la forme pédagogique du journal, médiation originale entre le gouvernement représentatif, dont on s'affirme le ferme défenseur par ailleurs; l'utopie de la "Nouvelle Atlantide" - ou gouvernement des experts ${ }^{9}$-, et la démocratie moderne, dont la liberté de la presse est l'une des conditions d'existence. Cette stratégie est indissociable de ce moment particulier qu'est la République Directoriale. Elle tend aux mêmes buts que la Société des Amis des Noirs et des colonies; société politique ouverte au débat d'idées, et société savante proposant des expertises pour une politique prospective. Encore quelques années, et l'évolution décrite par JeanLuc Chappey pour les sociétés savantes à l'époque consulaire ${ }^{10}$, à savoir une plus grande spécialisation et une quasi-exclusivité de la fonction d'expertise au détriment de la fonction publiciste, rendra caduque cette indifférenciation héritée de la sociabilité des Lumières. Les années de La Décade postérieures à 1802 correspondent d'ailleurs à un tout autre paradigme politico-idéologique, et n'ont pas été retenues dans cette étude.

5 Sachant donc que l'on peut parler d'un « moment » particulier dans lequel va s'inscrire le projet conservateur de La Décade, revenons à la question coloniale pour mieux apprécier en la matière les orientations majeures de la période. Les deux bornes chronologiques sont le décret du 16 pluviôse an II (4 février 1794), qui institue la liberté générale, d'une part, la loi du 30 floréal an X (20 mai 1802), qui la supprime et revient à la situation antérieure à la Révolution, d'autre part. Singulière volte-face historique, dont la brièveté (huit ans), ne saurait toutefois, sans contresens majeur, être interprétée comme une ellipse. Considérer que le rétablissement de 1802 était déjà inscrit dans l'émancipation de 1794, et considérer qu'il ne s'est rien passé d'important en la matière pendant ces huit années, relève de l'imposture intellectuelle ${ }^{11}$.

6 Les rédacteurs de La Décade qui vont s'exprimer sur la question coloniale à cette époque (1794 - 1802), sont les héritiers de la tradition encyclopédique. La réflexion sur les colonies s'ordonne alors autour de l'opposition entre les «colonies antiques » et les "colonies modernes». Cette dernière forme de colonisation, la «colonisation moderne ", fait l'objet d'appréciations contradictoires chez les penseurs des Lumières, 
tantôt condamnée sans nuances comme résultat d'une conquête prédatrice, à l'image de ce que fut le pillage de l'Amérique par les Espagnols, tantôt encensée comme génératrice $d u$ progrès économique et social ${ }^{12}$. Les termes de ce débat relèvent désormais de l'Histoire, depuis le décret d'abolition qui, pour ses partisans, fait davantage figure de principe que d'événement.

7 Dès lors, les rédacteurs de La Décade doivent conceptualiser la situation nouvelle; régénération des colonies modernes, et recherche de nouveaux établissements sur la base du principe de la liberté générale. C'est la « colonisation nouvelle ». La réflexion de La Décade, laboratoire d'idées, est parallèle aux trois grands textes qui scandent les orientations de la République en matière coloniale; le discours de Boissy d'Anglas du 5 messidor an III pour la régénération des colonies (Boissy propose l'assimilation); le discours de Talleyrand prononcé au cours des séances de l'Institut le 15 messidor an V (3 juillet 1797) sur la colonisation nouvelle (Talleyrand propose de se tourner vers l'Afrique) ; la loi du 12 nivôse an VI (1er janvier 1798) pour la départementalisation des Antilles. Ces trois textes majeurs, que l'on présente abusivement comme complémentaires ${ }^{13}$, reflètent en réalité des orientations divergentes. Mon propos n'est pas de faire l'analyse de ces textes, mais d'éclairer, à travers les articles de La Décade, le fond idéologique dont ils s'inspirent.

8 Remarquons encore les lieux où ces discours sont prononcés: la représentation nationale, l'Institut. Ces lieux illustrent la stratégie des réseaux d'influence développés par les milieux de La Décade. Il manque le Ministère de la Marine et des colonies. Truguet, ministre entre 1795 et 1797, républicain conservateur et abolitionniste intransigeant, était très proche des thèses développées par La Décade.

9 Les articles traitant de la question coloniale relèvent de diverses rubriques dans la présentation du journal. Trois regroupements ont été opérés.

10 - La partie événementielle. Elle apparaît comme secondaire, cette relégation des faits qui concernent l'évolution de la situation dans les colonies régénérées, s'explique par le postulat de base. L'abolition étant l'expression d'un principe, et non le produit des circonstances, tout événement qui contrarie le processus de régénération ne peut s'expliquer que par la persistance d'un état antérieur d'oppression, que le temps va progressivement effacer. Nous verrons que cette interprétation historicisante de la situation, à Saint-Domingue tout particulièrement, laisse les rédacteurs de La Décade totalement désemparés dans le contexte de 1801-1802.

11 - Les comptes rendus qui relèvent de la rubrique Voyages pourraient s'apparenter à la simple curiosité pour les peuples lointains, refléter l'évolution des sensibilités de plus en plus marquées par l'exotisme. En réalité, ils s'inscrivent dans une réflexion de fond, qui prend pour objet d'analyse le concept de « colonie nouvelle ».

12 - C'est donc cette réflexion, avec les comptes rendus d'ouvrages qui s'y rattachent, qui a retenu principalement mon attention. Il faut prendre en compte les auteurs des ouvrages analysés, mais aussi et à égalité, les auteurs des comptes rendus, privilégiant ainsi les liens entre les uns et les autres, pour reconstituer un "milieu". Parmi les auteurs de La Décade, reviennent les noms de Jean-Baptiste Say, de Charles-Guillaume Théremin, de Joachim Le Breton, de Pierre-Louis Ginguené, de Georges Toscan. Je les ai appréhendés dans ce moment particulier de la Première République, sans tenir compte des jugements postérieurs qui les rattachent ou les excluent du groupe des « Idéologues » au sens strict. La question est d'importance, pour quelqu'un comme Say, 
ou, à l'inverse, en ce qui concerne la présence silencieuse de Cabanis ; mais elle dépasse le cadre de cette contribution.

13 Les colonies qui sont restées dans l'orbite républicaine sont Saint-Domingue, progressivement soustraite à l'occupation anglaise, la Guadeloupe, la Guyane, l'île-deFrance (île Maurice), et l'île de la Réunion. Ces colonies sont les " colonies modernes ", selon la terminologie en vigueur; exploitées sur la base du système de l'économie de plantation, qui nécessite un constant renouvellement de la main-d'œuvre servile par la traite, elles symbolisent l'Ancien Régime. Elles ont amorcé un processus de régénération à partir de la proclamation de la liberté générale, réalisée sur l'initiative de Sonthonax et Polverel, commissaires à Saint-Domingue, le 29 août 1793, puis généralisée par le décret du 16 pluviôse an II (4 février 1794). Mais ce processus est chaotique, ensanglanté par tous les épisodes d'une guerre raciale qui recoupe plus ou moins confusément une guerre contre l'occupant britannique. Si l'importance de SaintDomingue dans ce qui reste de l'empire colonial français justifie le fait que l'ancienne «perle des Antilles » se taille toujours la part du lion dans la relation des événements coloniaux, on perçoit la gêne des rédacteurs lorsqu'il leur faut donner l'interprétation de ces événements.

14 Il y a tout d'abord la plus élémentaire prudence qui joue lorsqu'il s'agit de rendre compte des faits qui se déroulent à des milliers de kilomètres de la métropole, dans un contexte de blocus maritime, et de situation militaire mouvante ${ }^{14}$. Mais il y a plus : les circonstances mêmes qui entraînèrent la proclamation de la liberté générale rappellent beaucoup trop la Terreur, un régime totalement réprouvé par les républicains conservateurs de La Décade. Aussi vont-ils s'efforcer de décontextualiser le principe (la liberté générale), qu'ils approuvent par ailleurs. De telle sorte que la transcription des nouvelles qui parviennent de l'île est parcimonieuse, contradictoire, embarrassée.

L'action du commissaire Sonthonax est évaluée de façon confuse : tantôt les lecteurs sont mis au courant des conclusions du rapport de la commission qui innocente les responsables de la première mission ${ }^{15}$. Tantôt le journal se fait l'écho des dénonciations portées par les représentants du lobby des planteurs blancs ${ }^{16}$. Quitte à accorder ensuite une large place à la relation de sa seconde mission par le même Sonthonax ${ }^{17}$.

Les mêmes contradictions sont perceptibles au sujet de Toussaint-Louverture. Si le journal publie en fructidor an IV (août 1796) une longue lettre de l'ingénieur Giroud, qui fait un vibrant hommage des généraux de couleur ${ }^{18}$, l'évolution est de plus en plus critique au fil des ans, jusqu'à la reproduction des pièces officielles qui incriminent la "conduite perfide et cruelle de Toussaint et Christophe", pièces qui justifient l'expédition militaire de Leclerc, avec les tragiques conséquences que l'on sait ${ }^{19}$.

17 Les informations sur les autres colonies sont réduites à la portion congrue. On ne trouve aucune trace des événements survenus aux Mascareignes, lorsque les assemblées coloniales de ces îles déportèrent les commissaires Baco et Burnel, venus proclamer le décret du 16 pluviôse ${ }^{20}$.

18 La situation à la Guadeloupe n'est évoquée que pour reproduire un rapport de Victor Hugues, essentiellement consacré aux succès militaires remportés par les républicains aux petites Antilles ${ }^{21}$. Le grand débat sur les colonies au Conseil des Cinq-Cents, au printemps 1797, rencontre peu d'intérêt dans les colonnes de La Décade ${ }^{22}$, alors que la motion de Wilberforce devant le Parlement britannique, en vue d'obtenir l'abolition de l'esclavage, est signalée ${ }^{23}$. 

principe. C'est tout le sens d'un long article, dû à la plume d'Horace Say, placé à la rubrique "affaires intérieures»- dont $\mathrm{H}$. Say est responsable -, dans le $\mathrm{n}^{\circ} \mathrm{du} 20$ pluviôse an IV (9 février 1796):

«...de cette agrégation, à la fois monstrueuse et ruineuse, de deux contrées dont l'une est aux antipodes de l'autre, et d'un gouvernement situé à deux milles lieues des gouvernés. Ne reconnaîtra-t-on pas un jour l'abus du régime prohibitif dans le commerce? Ne l'abandonnera-t-on pas généralement? Et, dès lors, quel avantage un État pourra-t-il retirer de ses colonies? Aucun. Il ne resterait que les inconvénients de cette liaison: une correspondance longue, pénible, dépendante des vents et des flots; la dépense d'une armée navale ad hoc, la réunion sous une même loi d'hommes différents par les mœurs et le climat. Mais la révolution dont nous parlons ne peut pas être partielle; l'état qui se détacherait seul de ses colonies, les livrerait à ses ennemis, à ses rivaux, et se mettrait à leur merci. Conservons donc les nôtres; en attendant l'époque où, sans danger pour elles et pour nous, elles pourront être indépendantes... » ${ }^{24}$

Dans le numéro suivant, à la rubrique «Affaires étrangères ", le journal enfonce le clou, mais en dégageant de plus larges perspectives d'avenir, en reliant la disparition du système de la «colonisation moderne » au développement du système de la « colonisation nouvelle» :

«La question de base est: les colonies pourront-elles retourner à l'état de splendeur, et surtout d'utilité pour la mère-patrie, où elles étaient avant la Révolution?

Certains ne veulent même pas qu'il y ait un doute sur la réponse. Et, pourtant, le doute est raisonnable: les colonies se soutenaient par le travail servile. Le nègre, devenu libre, n'a plus aucun intérêt à soutenir cette prospérité: près de la nature, il ne sent aucun de ces besoins factices qui donnent une si grande activité à l'habitant de l'Europe vieille, policée et corrompue. Si une nourriture grossière, si une faible portion de manioc suffit au nouveau citoyen des Antilles pour satisfaire sa faim ; s'il n'a besoin que d'une chemise et d'un caleçon de toile pour couvrir sa nudité, ne regardera-t-il pas comme insensé celui qui voudrait l'engager dans des travaux qui doivent ruiner ses forces, l'arracher sans cesse d'auprès de sa compagne, en lui offrant, pour dédommagement, des mets que son palais grossier ne peut savourer, ou un habit dans le goût européen?

L'insurrection des nègres doit devenir générale dans les Antilles; aussi les anglais ont-ils pris les devants, et cherché à se procurer des établissements d'un type nouveau, comme la colonie de Sierra-Leone. L'esclavage n'y est pas introduit, la terre est sollicitée par le natif lui-même, les produits vont remplacer un jour prochain ceux de la Jamaïque. Il y a tout lieu de supposer par ailleurs que les anglais convoitent l'Egypte, qu'ils songent ainsi à verrouiller solidement la route des Indes. Il faut que les français comblent le retard qu'ils ont pris sur les britanniques.» ${ }^{25}$

Nous retrouvons là, avec un an et demi d'avance, l'essentiel de l'argumentation développée dans le rapport de Talleyrand devant l'Institut, le 3 juillet 1797. Ce mémoire a été rédigé par Desrenaudes, un proche de La Décade. Par ailleurs, le journal accorde toute son attention à l'œuvre de l'abbé Coisnon, un proche de Grégoire, qui a fondé une Institution Nationale des colonies dans les locaux de l'ancien Collège de la Marche. Il s'agissait de réunir, dans une institution d'enseignement privé, des élèves internes issus des familles dirigeantes des colonies, recevant une instruction commune sans 
aucune distinction de couleur ${ }^{26}$. On retrouve là les enfants de Toussaint-Louverture et d'autres généraux noirs, les enfants du général mulâtre Rigaud, le fils de Brissot, ainsi que des héritiers des familles de planteurs blancs, et même des membres de l'aristocratie militaire de la côte dalmate, etc. Cette école des cadres, qui a reçu la protection du ministre de la marine Truguet, est mentionnée avec éloges dans le numéro du 20 vendémiaire an VII (11 octobre 1798). Son existence sera brève toutefois. Le ministre de la marine Decrès vient brutalement y mettre un terme en octobre 1802, affirmant que les enfants de couleur ne peuvent prétendre à une éducation supérieure, au-delà de quelques rudiments des arts mécaniques ${ }^{27}$. La Décade relate solennellement la fin de l'expérience et la dispersion des élèves, tout en faisant un vif éloge de l'abbé Coisnon, manifestation (bien discrète) d'opposition à une mesure qui s'inscrit tout à fait dans le contexte rétrograde du printemps 1802.

"Ceux qui, comme nous, ont assisté ces dernières années à la distribution solennelle des prix et aux exercices qui la précédaient, sous les auspices et en présence du Ministre de la marine, savent avec quelle justesse et précision les élèves répondaient aux questions qui leur étaient faites, et avec quel vif enthousiasme ils étaient applaudis par les nombreux spectateurs qui les entouraient ${ }^{28}{ }^{28}$

24 L'attention portée par La Décade à cette expérience signale aussi la préférence accordée à l'évolution sur le long terme, au rôle central tenu par l'instruction publique pour la consolidation des institutions républicaines. Notons encore que cette expérience s'inscrit dans le cadre de la doctrine d'intégration républicaine, dont l'application la plus concrète fut la grande loi organique sur la départementalisation des colonies, du 12 nivôse an VI. On ne relève pourtant aucun compte rendu, aucune analyse de cette loi dans «La Décade». Tout se passe comme si l'effort de réflexion avait porté essentiellement sur le phénomène de la "colonisation nouvelle", alors que la loi entamait un véritable processus de décolonisation.

La «colonisation nouvelle » relève en fait d'un courant idéologique et d'une série d'expérimentations antérieurs à la Révolution française ${ }^{29}$. Les sociétés abolitionnistes anglaises jouèrent un rôle pionnier en la matière. Tout part de la critique de l'économie de plantation, qui est le pilier de la «colonisation moderne». Le postulat des économistes libéraux (Smith, Turgot, Dupont de Nemours) est la non-rentabilité de l'économie esclavagiste, en raison de la faible productivité de la main-d'œuvre servile ${ }^{30}$. La condamnation de l'esclavage ne reposait plus seulement sur des bases morales, et cette avancée permettait de dépasser la querelle des Anciens et des Modernes en matière de colonisation. Pour les encyclopédistes radicaux (tels que Diderot, Pechmeja, Damilaville [31]), la seule forme de colonisation admise était l'établissement agricole européen, en territoire vierge, ou très faiblement peuplé, mis en valeur par des colons cultivateurs développant un type de production proche de l'autosuffisance. C'est le type de mise en valeur qui se rapproche le plus de ce qu'on pense être la colonisation " antique » (ou " ancienne»); des lotissements égalitaires de colons agriculteurs, de colons vétérans, vivant quasiment en circuit fermé, pratiquant un échange minimum avec les populations indigènes ${ }^{32}$.

Les défenseurs de la "colonisation moderne " insistent au contraire sur l'économie d'échanges et l'augmentation des richesses et du bien-être induite par la généralisation de la consommation des produits «de luxe». Les libéraux abolitionnistes veulent convaincre en priorité les groupes professionnellement sensibilisés à ce type d'arguments (négociants, armateurs, banquiers), que la substitution graduelle du 
travail salarié au travail servile ne provoquera pas la ruine de l'économie de plantation, mais augmentera au contraire sa productivité.

Des doutes subsistent néanmoins; sur le long terme (les coûts de formation et d'encadrement ne risquent-ils pas d'obérer les gains de productivité espérés ?), aussi bien que sur la cohérence interne (la pensée libérale insistant fortement sur la notion d'«intérêt», peut-on attendre du travailleur une motivation forte dès lors que le produit de son travail continuera de lui être extérieur ?). Il faut alors envisager un type d'établissement radicalement nouveau, sur des terres non encore exploitées par les Européens, reposant sur un transfert de technologie avec les populations indigènes. $\mathrm{Ce}$ type d'échanges était déjà préconisé dans les instructions données aux officiers de marine qui voyageaient dans le Pacifique à la fin du XVIII siècle ${ }^{33}$. C'est surtout cette orientation qui est valorisée par La Décade.

Elle rejoint les préoccupations du jeune Jean-Baptiste Say, non seulement critique envers les postulats du libéralisme classique (la plus grande rentabilité du travail libre par rapport au travail servile [34]), mais encore soucieux de préserver et d'étendre les positions de la "Grande Nation» dans le monde, pour concurrencer la GrandeBretagne. Say met en valeur l'ouvrage du Suédois Charles-Bernard Wadström : Essay on colonization. Ce modèle néo-colonial a une terre de prédilection, l'Afrique.

C'est dans le $\mathrm{n}^{\circ}$ du 10 brumaire an IV que l'ouvrage de Wadström est signalé à la rubrique "Annonces ». Il n'est pas question de retracer ici la carrière de cet ingénieur suédois, passionnante par ailleurs ${ }^{35}$. Disciple de Swedenborg, émigré en Angleterre, il fréquenta les milieux abolitionnistes (Thomas Clarkson, William Wilberforce), et fut associé aux projets de «colonisation libre » du Sierra-Leone et de Bulama, dont nous reparlerons. Il se rend en France en octobre 1795, peu après les tragiques événements qui entraînèrent la destruction de l'établissement du Sierra-Leone par des navires français, en février $1795{ }^{36}$. Cette affaire est relatée par l'abbé Grégoire le 10 ventôse an IV (29 février 1796) ${ }^{37}$.

30 Les références swedenborgiennes de Wadström ne relèvent pas non plus de quelque résurgence des « brouillards du Nord ». À la rubrique «Philosophie », le 30 nivôse an VI (19 janvier 1798), un article rappelle que c'est aux swedenborgiens que l'on doit les initiatives, portées devant le Parlement britannique, pour abolir la traite et l'esclavage. Il précise que Swedenborg place au cœur du continent africain la «Nouvelle Jérusalem », et que l'établissement du Sierra-Leone est un point de départ pour des expéditions vers l'intérieur de l'Afrique :

« un point de départ pour l'exploration de l'intérieur du continent, afin d'en étudier les peuples et l'histoire naturelle, pour y porter les inventions utiles de l'Europe, les principes de la civilisation, les idées de morale. Il y a ainsi un but missionnaire; visiter les nations noires, prendre contact avec les rois, gagner leur confiance, et les engager, par le sentiment de l'humanité, tout autant que par celui de leur propre intérêt, à renoncer au trafic des esclaves. " ${ }^{38}$

Il n'est guère surprenant, dès lors, de voir figurer, à la rubrique « Voyages » des comptes rendus d'ouvrages tel que celui de Mungo Park sur la boucle du Niger, à la recherche de Tombouctou, identifiée à la « Nouvelle Jérusalem » ${ }^{39}$. Ou bien des études sur l'histoire des États africains, telle l'Histoire du Dahomey, d'Archibald Dalzel, un ancien gouverneur du comptoir de traite de Ouidah ${ }^{40}$.

Jean-Baptiste Say et Charles-Bernard Wadström sont également membres de la Société des Amis des Noirs et des colonies, dont j'ai publié les notes de séance entre décembre 
1797 et mars 1799. J'ai ainsi pu établir que Say (mais aussi son épouse), Wadström (et son épouse) figurent parmi les membres dirigeants de cette deuxième société abolitionniste, au même titre que Lanthenas, Benjamin-Sigismond Frossard, le général Servan, ou l'abbé Grégoire. Une lettre insérée dans le $\mathrm{n}^{\circ}$ du 20 floréal an VI - 9 mai 1798 - souligne la profonde continuité avec la Société qui existait au début de la Révolution, et précise les buts de la nouvelle; les Africains sont perfectibles, mais ils sont encore dans l'enfance. Ils « ignorent tout le parti qu'ils pourraient tirer de leur sol et de leur climat». Il faut donc opérer un transfert de technologie en les plaçant dans une relation pédagogique, où l'Européen est l'instructeur. «Ils ont un besoin urgent d'instructions morales et physiques. Ils font preuve d'une grande aptitude pour tous les arts, mais il faut leur en apprendre les éléments, la marche et la pratique». Il y a transfert de l'idéal missionnaire à l'idéal pédagogique. Mais la relation anthropologique reste identique ; l'Européen, et spécialement le Français, est devenu l'instituteur des autres mondes.

La lettre propose ensuite, à titre d'exemple, un mémoire sur la culture de la canne à sucre à Batavia (capitale des Indes néerlandaises), qui est l'œuvre du général Servan ${ }^{41}$. Ce mémoire insiste sur le progrès technologique, qui permet de produire le rhum et le sucre à un bien meilleur marché que dans les Antilles, tout en économisant le travail humain. Ce qui démontre également la supériorité du travail libre sur le travail servile. Il faudrait substituer la charrue à la houe, qui est encore l'instrument de base aux Indes occidentales; développer les pâturages pour la traction animale et l'autosuffisance alimentaire; concentrer les entreprises de distillation, au lieu d'en avoir une par exploitation; développer le système hydraulique de Montgolfier et Argand pour l'irrigation, plus efficace et plus sain que l'irrigation par canaux.

Nous connaissons par ailleurs l'attention portée par La Décade à tout ce qui concerne le progrès agronomique; les préoccupations sont les mêmes que celles de nombreux mémoires techniques présentés à la Société des Amis des Noirs et des colonies. De nombreuses propositions sont faites pour l'amélioration de l'alimentation, en particulier par Lasteyrie du Saillant ${ }^{42}$, qui envisage une production de farine de pomme de terre; ou bien Giroud, l'ingénieur déjà cité, qui propose l'extension de la culture du fameux arbre à pain ${ }^{43}$. Tous deux sont membres de la Société des Amis des Noirs et des colonies.

Ainsi, la symbiose est totale entre une partie de la rédaction de La Décade et une partie influente du groupe dirigeant de la Société des Amis des Noirs. Le journal sert de caisse de résonance pour les ouvrages des membres de la Société, comme le philanthrope Charles Montlinot, qui écrit un Essai sur la transportation comme récompense et la déportation comme peine. Le compte rendu est signé par un autre membre de la Société, Charles Théremin ${ }^{44}$. Il se termine par un hommage appuyé à Wadström:

«Un voyageur Suédois (Mr. Wadström), qui a résidé longtemps dans les établissements anglais de Sierra-Leone, et qui connaît par lui-même une partie considérable des côtes de l'Afrique, a écrit un excellent Essai sur la colonisation; il est rempli des mêmes vues philanthropiques que l'ouvrage de notre auteur. M. Wadström veut, comme lui, civiliser les nègres au lieu de les acheter et de les vendre; il propose de former sur les côtes d'Afrique une colonie européenne qui puisse remplir ce but, et enseigner aux africains la culture de la terre, et les principaux arts mécaniques; il partirait lui-même avec ses colons et dirigerait l'établissement $[. .]$. 
parachever cette description d'un "milieu", et illustrer le fonctionnement d'un réseau d'influence, à travers la circulation des textes, repartons de l'article nécrologique sur Wadström, paru dans le $n^{\circ}$ du 10 floréal an VII (29 avril 1799) de La Décade. Cet article se présente sous forme d'une « lettre de la citoyenne Hélène-Maria Williams au citoyen Jean-Baptiste Say sur la mort du philanthrope Wadström» (Wadström est mort à Paris d'une consomption pulmonaire le 15 germinal an VII). Hélène-Maria Williams, par ailleurs figure éminente de la seconde Société des Amis des Noirs, a écrit plusieurs ouvrages, traduits par Jean-Baptiste Say, dont les comptes rendus élogieux ont été publiés dans La Décade. Elle est également la compagne de JohnHurford Stone, qui eut des participations dans la Compagnie de Sierra-Leone et qui figure aussi parmi les membres dirigeants de la Société des Amis des Noirs, ami de Talleyrand et d'Horace Say, etc. ${ }^{45}$.

37 J'ai ainsi voulu rendre manifeste l'importance et la cohérence d'un projet baptisé " colonisation nouvelle", pour le courant républicain conservateur. Il faudrait aussi, dans un registre différent, mais voisin, et sans apparente solution de continuité, mentionner l'appui résolu apporté par les frères Say, Stone, Le Breton et autres, à l'expédition d'Égypte.

Est-ce à dire que la proposition des "établissements libres » épuise la question coloniale ? Certainement pas. Les positions des tenants de la « restauration coloniale » d'Ancien régime, qui se déchaînent au printemps 1797, et qui finissent par l'emporter au printemps 1802 , sont à peine mentionnées ${ }^{46}$. Il est vrai que la polémique n'est pas la caractéristique du journal, qui se veut force de proposition et de réflexion, et non de contestation et d'action, comme le sont les organes d'opinion crypto-royalistes ou néojacobins.

à l'intérieur même de la Société des Amis des Noirs, autour de Sonthonax, de Laveaux, et de l'essentiel de la députation de Saint-Domingue, très impliquée, elle, dans l'évolution des événements aux Antilles, existe un courant d'idées, lié prioritairement à l'opposition démocratique, davantage tourné, par nature et par fonction, vers l'approfondissement de la "régénération républicaine» et du statut futur des " colonies modernes ", soustraites à la menace anglaise ${ }^{47}$.

Quelques échos de ces préoccupations se retrouvent dans La Décade. Concernant tout d'abord le statut social des " nouveaux libres ${ }^{48}$, le maintien du cadre de l'économie de plantation ne va pas de soi pour Édouard Lefebvre, membre de la Société Libre des sciences, belles-lettres et arts de Paris, auteur d'un ouvrage intitulé Considérations politiques et morales sur la France constituée en république. Un compte rendu détaillé est paru à la rubrique « Politique raisonnée » du 30 vendémiaire an VII (21 octobre 1798). Il est dû à Vieilh de Boisjolin. Le chapitre XVII traite « des colonies »; Lefebvre préconise une véritable réforme (révolution?) agraire, qui briserait le carcan de la plantation. Pour établir conjointement le travail et la liberté, "il faut que le nègre soit propriétaire ». Il doit recevoir autant de terres que ce qui sera nécessaire à la subsistance de sa famille, terres prises sur les héritages coloniaux abandonnés par les planteurs. À terme, l'auteur préconise une immigration européenne, afin de généraliser le savoir-faire technique, mais aussi pour rompre avec l'idée reçue selon laquelle les Européens seraient inaptes au travail manuel sous les climats tropicaux ${ }^{49}$. La régénération s'appuie enfin sur une promotion systématique des mariages mixtes. Il ne faut pas hésiter à doter des femmes noires, veuves ou célibataires, d'un lopin de terre. Ainsi, elles n'auront aucune peine à fonder des unions stables avec les petits Blancs.

Annales historiques de la Révolution française, 339 | janvier-mars 2005 
D'autres projets de régénération sont élaborés, avec le soutien actif de la députation de Saint-Domingue. Ainsi, la loi de départementalisation du 12 nivôse an VI est-elle l'aboutissement d'une lutte opiniâtre menée par ces représentants contre les partisans d'une restauration de l'ordre colonial traditionnel, sous couvert d'autonomie locale. Le principal inspirateur de cette loi, le général Laveaux, ex-gouverneur de SaintDomingue, parle des cultivateurs noirs comme des " véritables colons", reconnaissant en ces derniers les propriétaires authentiques du sol antillais, selon un statut qui n'est guère éloigné de celui préconisé par Édouard Lefebvre. Laveaux renoue ainsi avec la tradition encyclopédique qui n'admettait comme forme de colonisation légitime que la « colonisation ancienne » (mise en valeur du sol par un colonat de petits propriétaires). $\mathrm{Au}$ cours de la discussion sur cette loi, un autre général, membre de l'Institut, Lacuée, propose que l'on supprime l'équivoque, et que l'on cesse de désigner les anciennes possessions ainsi régénérées par le terme de "colonies». Le terme de remplacement serait celui de "départements d'outre-mer ", ou d'" établissements d'outre-mer ", appellation que l'on trouve fréquemment aussi dans les discours de Laveaux ${ }^{50}$. La justification théorique de ce glissement sémantique est empruntée implicitement à Destutt de Tracy:

"Comme il n'est aucun de vous, citoyens collègues, qui ne soit convaincu que les langues, en changeant les signes, modifient les idées, que les têtes se forment sur les langues, et que les pensées prennent la teinte des idiomes [...] » Nous avons là une démonstration de " politique raisonnée ».

Toutefois, ces positions inspirées d'un individualisme égalitaire radical, ne semblent guère prisées par La Décade, qui ignore, à l'exception notable de Lefebvre, les projets de régénération des "colonies modernes». L'évolution de la situation aux Antilles, et notamment la promulgation par Toussaint-Louverture d'une constitution garantissant les situations acquises par la nouvelle bourgeoisie noire, en 1801, ouvre des perspectives nouvelles. À cet égard, le compte rendu de l'ouvrage de l'abbé de Pradt, Les trois âges des colonies, marque un tournant ${ }^{51}$.

Rappelons que l'ouvrage de l'abbé de Pradt est construit de façon paradoxale; après deux parties où il s'efforce de montrer que les "colonies modernes" reposent sur l'économie de plantation, et que cette dernière ne peut perdurer sans l'esclavage, il va démontrer dans une dernière partie que, puisque l'esclavage est aboli, il est vain de vouloir restaurer l'ordre ancien. Aussi l'auteur souhaite que l'on s'achemine vers l'indépendance, avec l'aide des anciennes métropoles. Ce souhait s'accompagne d'un éloge appuyé de la nouvelle bourgeoisie noire. La tourmente révolutionnaire a permis de révéler des hommes de grands talents, particulièrement dans l'armée et dans l'administration :

«le plus renommé de ces chefs, le nègre Toussaint-Louverture, semble destiné à être le Washington des colonies. Son caractère paraît grand; il a montré une élévation de sentiments qui l'honore, des talents qui le rendent recommandable: c'est le chef le plus fort et le plus accrédité de tous les noirs. »

Et, plus loin, il révèle le sens véritable de cette résignation à l'inéluctable:

«...comment effacer de leur souvenir le temps où ils furent libres, dominants, vainqueurs de ces mêmes blancs qui voudraient les maîtriser de nouveau [...] ? ${ }^{52}$

Ce compte rendu date du 10 nivôse an X (1er janvier 1802). Une quinzaine de jours auparavant, l'expédition Leclerc a quitté Brest. À sa manière, distanciée, $L a$ Décade exprime son désarroi face à un événement dont la signification profonde lui échappe. 
Le système d'indépendance noire de l'abbé de Pradt est certainement une utopie, pourtant, c'est « une illusion bienveillante [...] Nous avons assez fait connaitre son plan pour lui obtenir de l'estime » ${ }^{53}$. Comme pour l'éloge de l'abbé Coisnon un peu plus tard, au moment de la fermeture de l'Institut National des colonies, la critique est indirecte ; ces hommes d'“influence" doivent se comprendre à demi-mot.

Mais la distance est aussi le signe d'un profond désarroi. Le parti-pris de décontextualisation du principe de la liberté générale, par souci de l'extraire du processus révolutionnaire ( des événements qu'on n'avait pu ni prévoir, ni calculer, précipitèrent la marche de la Révolution, et les noirs devinrent entièrement libres au moment où l'on n'osait à peine espérer de voir diminuer le poids de leurs chaînes ", rappelaient les Amis des Noirs dans leur lettre aux rédacteurs de La Décade du 20 floréal an VI) s'est révélé vain, puisque l'événement a fini par rattraper et déstabiliser l'institution.

Il faut maintenant admettre que brumaire a été une journée des dupes. La Constitution libérale de 1795, qui fut largement l'œuvre des républicains conservateurs, a été sacrifiée par ceux-ci en invoquant la sauvegarde des institutions (selon Horace Say, «la constitution administrative est plus importante pour le bonheur des nations que la constitution politique » $\left.\left.{ }^{54}\right]\right)$. Or, voilà que le nouveau Cincinnatus, loin de retourner à sa charrue, remet en cause ces mêmes institutions, en commençant par détruire la liberté générale. Au sein du Tribunat préalablement épuré, le 29 floréal an X (19 mai 1802), il y eut encore vingt-sept opposants au rétablissement de l'esclavage, pour cinquante-quatre qui approuvèrent ${ }^{55}$. La résistance n'est pas négligeable. Si Ginguenné, Boisjolin, Andrieux, Desrenaudes avaient été épurés, on trouvait encore nombre de tribuns qui s'étaient distingués par leurs prises de position abolitionnistes (JeanBaptiste Say, Joachim Le Breton, Joseph Eschassériaux, notamment). Les historiens ${ }^{56}$ font valoir que les Idéologues auraient été l'âme de cette résistance tribunitienne. Le vote se déroulant dans l'anonymat, on en est réduit aux suppositions et à l'analyse des parcours individuels, ce qui serait l'objet d'une autre communication.

\section{NOTES}

1.Joanna KiTcHIN, Un journal philosophique: La Décade : 1794 - 1807, Bibliothèque de littérature et d'histoire $n^{\circ}$ 5, Lettres modernes, 1965.

2.Marc Regaldo, Un milieu intellectuel; La Décade philosophique: 1794 - 1807, Paris, Honoré Champion, 1976, 5 volumes.

3.Éditions La Découverte, 1992 (tout particulièrement les pp. 234 à 240).

4.Éditions Karthala, 1998.

5.La Société des Amis des Noirs, 1788 - 1799, Paris, Éditions de l'Unesco, collection «la route de l'esclave», 1998.

6.Françoise BRUNEL, Thermidor, la chute de Robespierre, Bruxelles, Éditions complexe, 1989, 2e partie: «Identifier le peuple à sa constitution», pp. 43-71. La thématique des «institutions républicaines» y est enracinée dans le projet montagnard de stabilisation 
du printemps de l'an II. Je reste pourtant persuadé que cette thématique, dans sa complémentarité antagonique avec celle de la Constitution, est centrale pour l'ensemble de la Première République; elle ne serait donc réductible ni à un «moment» particulier (le printemps 1794), ni à un courant politique circonstanciel (les Montagnards). Je renvoie pour illustrer ce point de vue à la brochure de Benjamin Constant, de 1796, De la force du gouvernement actuel de la France, et de la nécessité de s'y rallier.

7.Ce qui peut expliquer, entre autres, le ralliement de ce courant «républicain conservateur» à Brumaire, au nom de la défense des institutions républicaines. Qu'il y ait eu alors «journée des dupes» est une toute autre affaire...

8.Thierry LENTZ, «La presse républicaine modérée sous la Convention thermidorienne et le Directoire ; Pierre-Louis Roederer, animateur et propriétaire du Journal de Paris et du Journal d'Economie politique », Revue Historique, n 592 (1994), pp. 297-313.

9.C. COUTEL, «Utopie et perfectibilité : significations de l'Atlantide chez Condorcet», dans Condorcet, homme des Lumières et de la Révolution, actes du colloque Recherches nouvelles sur Condorcet, ENS Éditions, 1997, pp. 99-107. Le texte de Condorcet : Fragments sur l'Atlantide, ou efforts combinés de l'espèce humaine pour le progrès des sciences renvoie à l'édition Garnier-Flammarion, pp. 299-348.

10.Jean-Luc CHAPPEY, « Les sociétés savantes à l'époque consulaire », Annales historiques de la Révolution française, 1997, pp. 451-472. Et, plus largement, du même : La Société des Observateurs de l'Homme, 1799 - 1804. Des anthropologues au temps de Bonaparte, Paris, Publications de la Société des études robespierristes, 2002.

11.Telle est la nouvelle «vulgate», largement développée au moment du bi-centenaire de la première abolition de l'esclavage, en 1994, dans certains articles de revues à grand tirage, et de journaux d'habitude mieux informés (Louis SALA-MOLINS, « Il y a deux cents ans, l'abolition de l'esclavage », Le Monde du 20 - 21 février 1994). Le résultat fut bien souvent que, en 1998, on commémora le cent-cinquantenaire de l'Abolition de l'esclavage, en ignorant généralement - par inculture ou mauvaise foi - la législation révolutionnaire de la Première République.

12. Les deux opinions se trouvent dans le fameux ouvrage de l'abbé RAYNAL; Histoire philosophique et politique du commerce et des établissements des Européens dans les deux Indes, première édition en 1772, extraits choisis et présentés par Yves Bénot aux Éditions François Maspéro, 1981. Une réédition intégrale doit paraître en 2005, à la Bibliothèque des Introuvables. Sur cette question des théories de la colonisation, voir Yves BÉNOT, $L a$ Révolution française et la fin des colonies, op. cit., 2e partie ; et, du même: Diderot, de l'athéisme à l'anti-colonialisme, Paris, Éditions François Maspéro, 1970.

13.Le texte de Boissy est parfois présenté comme symbolique, à lui seul, de toute la politique du Directoire en matière coloniale ( Florence GAUTHIER, Triomphe et mort du droit naturel en Révolution 1789 - 1795 - 1802, Paris, PUF, 1992). Or, l'inspiration égalitaire de la loi du 12 nivôse an VI est radicalement autre que les présupposés "différentialistes" de Boissy. Voir Bernard GAINOT, « La naissance des départements d'outre-mer; la loi du 1er janvier 1798 ", Revue d'histoire des Mascareignes et de l'Océan Indien, 1/1998, pp. 51-74.

14.Un seul exemple, parmi de nombreux autres: le décret du 16 pluviôse an II a été promulgué à Cayenne le 6 thermidor an II (24 juillet 1794). Mais cette nouvelle n'est donnée que dans le $\mathrm{n}^{\circ}$ du 10 brumaire an IV (1er novembre 1795) de La Décade! 15.Rapport sur les troubles de Saint-Domingue, fait au nom des Comités de salut public, de législation et de marine réunis, 4 volumes, Paris, an $\mathrm{V}$, le principal rédacteur est J. Ph. 
Garran-Coulon. Ce rapport volumineux innocente entièrement les commissaires civils Polverel et Sonthonax (annonce dans le $n^{\circ}$ du 10 thermidor an VII [28 juillet 1799]). Un article, inséré dans le $\mathrm{n}^{\circ}$ du 20 vendémiaire an III (11 octobre 1794) avait rendu compte de la constitution de cette commission.

16. $\mathrm{N}^{\circ}$ du 20 nivôse an $\mathrm{V}$ (9 janvier 1797), puis $\mathrm{n}^{\circ}$ du 30 prairial an $\mathrm{V}$ (18 juin 1797). 17. $\mathrm{N}^{\circ}$ du 30 pluviôse an VI (18 février 1798).

18. «L République n'a pas d'amis plus sincères, de plus braves défenseurs, que ces hommes d'origine africaine que la France a récemment admis au nombre de ses enfants [...] plus tranquilles aujourd'hui sur leur chère liberté, ils embrassent avec franchise et transport leurs vieux amis Sonthonax et Raymond. Ils bénissent tous ces bons blancs qui ont écrit, combattu, et souffert pour leur liberté. ", $\mathrm{n}^{\circ}$ du 10 fructidor an IV (30 avril 1796).

19. $\mathrm{N}^{\circ}$ du 10 floréal an X (20 mai 1802).

20.Claude WANQUET, op. cit., pp. 283-310.

21. $\mathrm{N}^{\circ}$ du 30 thermidor an III (17 août 1795).

22. $\mathrm{N}^{\circ}$ du 30 prairial anV (cf. note 16). Sur ce débat et ses enjeux, Bernard GAINOT, «Bottu, le républicain des colonies », Annales historiques de la Révolution française, 293-294, 1993, pp. 431-444.

23. $\mathrm{N}^{\circ}$ du 20 germinal an IV ( 9 avril 1796). L'information est accompagnée de commentaires.

24.L'article est intitulé : « sur l'absurdité du système des colonies modernes ». 25.Cet article, intitulé « Réflexion sur l'état des colonies dans les Indes occidentales, et sur la nécessité de former ailleurs d'autres établissements », est en fait la reproduction d'un article de la Gazette Nationale de France ( ${ }^{\circ}$ du 30 pluviôse an IV - 19 février 1796). 26.Bernard GAINOT, « Un projet avorté d'intégration républicaine ; l'institution nationale des colonies (1797 - 1802) », Dix-huitième siècle, 32/2000, pp. 371-401. Compterendu de l'exercice public fait par les élèves noirs, sang-mêlés et créoles blancs à l'Institution Nationale des colonies, ci-devant Collège de la Marche, le cinquième jour complémentaire de l'an VI. Ce compte-rendu est accompagné d'un vif éloge de l'abbé Coisnon.

27.A.N., AF III 210, dossier 963 . Voici comment Blaise Lechat, un ancien élève noir, relate la fin de l'expérience dans une lettre à ses amis Placide et Isaac Louverture, datée de Marseille, 10 juillet 1814: « Le ministre Decrès vint à l'Institution, fit réunir dans la cour tous les américains (c.a.d. les élèves de couleur) et leur tint un discours très dur. Le gouvernement ne veut plus se charger de leur éducation, il en a déjà trop fait pour des êtres comme nous ".

28. $\mathrm{N}^{\circ}$ du 20 fructidor an X (7 septembre 1802).

29.Marcel DorignY, « La Société des Amis des Noirs et les projets de colonisation en Afrique ", Annales historiques de la Révolution française, $n^{\circ} 293$ - 294, spécial Révolution aux colonies, 1993, pp. 421-429.

30.Ph. STEINER, « L'esclavage chez les économistes français: 1750 - 1830 », dans Les abolitions de l'esclavage de Sonthonax à Schoelcher, actes du colloque de Paris VIII - SaintDenis (Unesco, 1995). Et L'économie de l'esclavage colonial. Enquête et bilan du XVII au XIX siècle (Fred CÉLIMÈNE et André LEGRIS, dir.), Paris, Éditions du CNRS, 2002.

31.Yves BÉNOT, La Révolution française et la fin des colonies, op. cit.

32.Tel est encore le modèle historique développé par Bouchaud, dans le $\mathrm{n}^{\circ} \mathrm{du} 30$ vendémiaire an VII sur « la formation des colonies romaines et des municipes » (Notice des travaux de la classe des sciences morales et politiques).

33.Jean-François de LA PÉrousE, Voyage autour du monde sur l'Astrolabe et la Boussole, Paris, Éditions La Découverte, 1980, pp. 25-38. 
34.Ph. STEINER, « Jean-Baptiste Say et les colonies, ou comment se débarrasser d'un héritage intempestif ", dans Cahiers d'économie politique, n²7-28, Éditions L'Harmattan, 1996. R. ARENA, « Pourquoi et comment faut-il abolir l'esclavage dans les Antilles françaises ? A propos des argumentations de Say, Sismondi et Rossi », dans L'économie de l'esclavage colonial, op. cit., pp. 73-84.

35.G. NIORD, Carl-Bernhard Wadström, de la Nouvelle Jérusalem à la seconde Société des Amis des Noirs, mémoire de maîtrise soutenu à l'université de Paris VIII-Saint-Denis, sous la direction de Marcel Dorigny (1997).

36.Yves BÉNOT, La démence coloniale (...), op. cit., pp. 158-159.

37. N Notice sur la Sierra-Leone et sur une calomnie répandue à son sujet contre le gouvernement français ».

38. "Quelques détails sur les disciples de Swedenborg, tirés du Voyage en Suède de M. Lenz de Schnepfenthal, effectué dans les derniers mois de l'année 1796 ».

39.Voyages et découvertes dans l'intérieur de l'Afrique, par le major Houngton et Mungo Park, ( $\mathrm{n}^{\circ}$ du 20 nivôse an VII - 9 janvier 1799). Ce compte rendu est dû à Joachim Le Breton. L'ouvrage a fait l'objet d'une édition récente aux Éditions La Découverte (1980 et 1996) ; voir en particulier les instructions données à Mungo Park par la Société africaine de Londres au sujet de la reconnaissance de la ville de Tombut (Tombouctou), p. 35. 40. $\mathrm{N}^{\circ}$ du 20 nivôse an IV - 20 janvier 1796 ; le titre exact est The history of Dahomey, an inland kingdom of Africa.

41.Les notes de séances de la Société des Amis des Noirs et des colonies nous apprennent que ce mémoire est extrait de Essay on colonization de Wadström. Servan le met en forme et le présente à la séance du 20 nivôse an VI (9 janvier 1798). Pour de plus amples informations, cf. Bernard GAINOT et Marcel DoRIGNY, La Société des Amis des noirs, op. cit.

42. $\mathrm{N}^{\circ}$ du 10 floréal an VI - 29 avril 1798 - ; rubrique « Économie domestique », Moyen de réduire les pommes de terre en farine.

43. $\mathrm{N}^{\circ}$ du 20 thermidor an V - 7 août 1797 - ; « De l'arbre à pain et de sa transformation en France et dans nos colonies ». Sur Giroud, cf. Robert CHAGNY, " Au temps de l'esclavage et de son abolition: planteurs grenoblois à Saint-Domingue » dans Évocations 1998/1999, pp. 83-116.

44.Sur Charles Théremin, cf. Sydney Seymour BIRO, The German policy of French Revolution, vol. 1, Cambridge, Massachussetts, 1957. L'article de Théremin sur Wadström se trouve dans le $n^{\circ}$ du 10 messidor an V - 28 juin 1797, à la rubrique « Législation ». 45.L. WOODWARD, Une adhérente anglaise de la Révolution française: Hélène-Marie Williams et ses amis (Thèse pour le doctorat de lettres, 1930).

46.Si l'on excepte le curieux ouvrage signalé dans le $\mathrm{n}^{\circ}$ du 10 messidor an V (28 juin 1797); De la nécessité d'adopter l'esclavage en France $1^{\circ}$ ) comme moyen de prospérité pour nos colonies $2^{\circ}$ ) comme punition pour les coupables $3^{\circ}$ )comme ressource en faveur des indigents. Le titre se suffit à lui-même... En 1797, l'auteur d'un programme esclavagiste est encore contraint à l'anonymat; après brumaire, il n'en sera plus de même. L'ouvrage est intéressant dans la mesure où il énonce une série de clichés racistes et esclavagistes que les abolitionnistes s'emploieront à réfuter au cours des années, voire des décennies suivantes.

47.Bernard GAINOT, « La députation de Saint-Domingue au Corps Législatif $\mathrm{du}$ Directoire ", dans Léger-Félicité Sonthonax. La première abolition de l'esclavage. La Révolution française et la Révolution de Saint-Domingue, $\mathrm{n}^{\circ}$ spécial de la Revue française d'histoire d'outre-mer, 316/1997, pp. 95-110. 
48.S. MANIGAT, «Qu'est-ce que la liberté générale en 1793 ? Les nouveaux libres en quête d'un projet..», Annales historiques de la Révolution française, « spécial colonies », op. cit., pp. 363-372; et aussi Yves BÉNOT, La Guyane sous la Révolution, ou l'impasse de la révolution pacifique, Kourou, Éditions Ibis rouge, 1997, pp. 64-79. 49. Cette idée est notamment longuement développée dans l'ouvrage anonyme mentionné à la note 46 sous le titre: « Peut-on espérer de faire fleurir nos colonies sans avoir recours à l'esclavage des nègres?».

50.Sur les termes de ce débat, et pour de plus amples informations concernant ce débat sur les termes, je renvoie à mon article paru dans La revue des Mascareignes, « La naissance des départements d'outre-mer... », op. cit., note 13.

51.Je renvoie à l'analyse critique du compte rendu de Joachim Le Breton par Yves BÉNOT, La démence coloniale (...), op. cit., pp. 272-278.

52. $\mathrm{N}^{\circ} \mathrm{S}$ du 10 nivôse an X (1er janvier 1802) et du 20 nivôse an X (10 janvier 1802). 53. $\mathrm{N}^{\circ}$ du 20 nivôse an X (11 janvier 1802); fin du compte rendu de Le Breton.

54.H. SAY, Sur les gouvernements libres et le degré d'obéissance qu'ils peuvent obtenir des peuples; compte rendu par J.-B. SAY, cité par M. Regaldo, op. cit.

55.A.N., C* I/ 165.

56.L. VILlEFOSSE et J. BouISSOUNOUSE, L'opposition à Napoléon, Paris, 1969, pp. 177-185.

\section{RÉSUMÉS}

La réflexion sur la colonisation à l'époque du Directoire s'inscrit dans les perspectives nouvelles ouvertes, tant par le décret d'abolition de l'esclavage du 16 pluviôse an II, que par la Constitution de l'an III qui place cette abolition au fondement des rapports nouveaux entre la métropole et ses colonies.

Héritiers d'une riche tradition encyclopédique en la matière, les rédacteurs du journal La Décade philosophique condamnent sans équivoque ce qu'ils appellent la « colonisation moderne » fondée sur le droit de conquête et l'exploitation de la main d'œuvre servile. Ils esquissent toute une réflexion sur les contours de la " colonisation nouvelle », un projet d'établissements européens situés essentiellement en Afrique, qui chercheraient à établir des échanges avec les populations indigènes sur la base du travail libre et des transferts technologiques.

Ce projet n'est pas exempt de contradictions et d'interrogations; outre la lancinante question du rapport des peuples indigènes au procès de civilisation, il traduit les hésitations de ses promoteurs sur la place des colonies «modernes» (Antilles, Mascareignes) dans la République ; quel degré d'émancipation politique? Quel avenir pour l'économie de plantation?

La Décade and the «New Colonization». Reflections on colonization during the Directory are bound to focus on the new prospects opened up by the decree abolishing slavery of 16 Pluviôse Year II and the Constitution of Year III which placed abolition at the core of the new relationship between the mother country and her colonies. Heirs to a rich encyclopedic tradition in this sphere, the editors of the journal La Décade philosophique were unanimous in condemning what they called «modern colonization» based on the right of conquest and slave labour. They charted the development of «new colonization», a scheme to set up European outposts situated mainly in Africa and aimed at forging trading links with the native population based on 
voluntary labour and technological transfers. The scheme was not without contradictions and question marks; apart from the invidious issue of the relationship between native peoples and the civilized world, it reflected the misgivings of its promoters on the role of «modern» colonies (the Antilles and Mascarene islands) within the Republic: what degree of political emancipation? What future did the plantation economy hold in store?

INDEX

Mots-clés : esclavage, Saint-Domingue, idéologues, abolitionnisme, colonisation, Sierra-Leone

\section{AUTEUR}

\section{BERNARD GAINOT}

Institut d'histoire de la Révolution française

Université Paris I Panthéon-Sorbonne

17 rue de la Sorbonne

75231 Paris cedex 05 